practice is lacking a validated, reliable tool with which to measure these kinematics. This study aimed to determine the accuracy of clinical gait analysis, by investigating concurrent validity, intra- and inter-rater reliability of two-dimensional (2D) video.

Materials and methods 21 participants with PFP were recruited (10 males, 11 females). Synchronised three-dimensional (3D) and 2D kinematic data were collected during over-ground running. 2D videos were analysed with the Hudl Technique application using a commercially available tablet (iPad). Single measure ICCs were calculated using a two-way mixed effects model with absolute agreement. 3D peak hip internal rotation (HIR) was investigated as a covariate with backward linear regression, using the $\mathrm{F}$ change statistic.

Results There was poor agreement between $3 \mathrm{D}$ and $2 \mathrm{D}$ measurement of peak HADD (ICC 0.06) and peak KFLEX (ICC 0.42). Moderate intra-rater reliability was identified for both variables (ICC 0.61-65). Inter-rater reliability for peak KFLEX was moderate (ICC 0.71), but was poor for peak HADD (ICC 0.31). 3D peak HIR did not significantly explain the identified poor agreement for either variable.

Conclusion Poor correlation between 3D kinematics and 2D video was identified for both variables in runners with PFP, despite acceptable intra-rater reliability. Investigation of software with increased precision is warranted, to improve the accuracy of $2 \mathrm{D}$ video predicting $3 \mathrm{D}$ kinematics in the clinical setting. Clinical gait analysis using the Hudl Technique application is not currently advocated.

\section{A NEW PROTOCOL FOR SONOGRAPHIC EVALUATION OF MID-PORTION ACHILLES TENDINOPATHY: A TEST RETEST INTRA AND INTER-RATER RELIABILITY STUDY}

${ }^{1}$ MSc Zubair Haleem*, ${ }^{2}$ Stephen Kelly, ${ }^{2}$ Dev Pyne, 'Simon Lack, ${ }^{1}$ Dylan Morrissey. ${ }^{1}$ Sports Exercise Medicine Dept - QMUL, Queen Mary University of London, UK; ${ }^{2}$ Rheumatology Dept, Mile End Hospital, UK

\subsection{6/bjsports-2019-scandinavianabs.30}

Introduction Achilles tendinopathy (AT) is common and recalcitrant. A new ultrasound Achilles tendon assessment protocol has been developed as part of a large RCT. Ultrasound scanning has considerable operator dependence hence this study was designed to investigate inter-rater and intra-rater reliability in symptomatic individuals.

Method Twenty-three participants (18 with AT, 10 female) were recruited and independently examined by two clinicians of varied experience. Measures of Achilles thickness, neovascularity, plantaris position and size, intratendinous tears (ITT) presence, fascia crura tear (FCT) presence, calcification, insertional elements and ultrasound provocation tests were recorded. Inter-rater and intra-rater reliability was assessed using Intraclass correlation coefficients (ICC), Standard error measurements (SEM) and minimal detectable changes (MDC) along with percentage agreements.

Results Excellent levels of inter- and intra-rater reliability were found for thickness (Intra-rater ICC 0.88-0.99, SEM 0.150.37 , Inter-rater ICC $0.87-0.98$ SEM 0.22-0.60), neovascularity (Intra-rater ICC $0.96-0.96$, SEM 0.28-0.40, Inter-rater ICC $0.91-0.94$ SEM $0.49-0.50$ ) and $100 \%$ agreement on insertional findings, ITT, FCT and US provocation tests. 67\%91\% agreement was found on presence, position and size of plantaris.
Conclusions The new protocol demonstrates excellent interand intra-rater reliability thus minimising operator dependence and is suitable for objective assessment. Further development of methods to identify the presence, position and size of the plantaris tendon is recommended.

\section{RISK FACTORS FOR LOWER-EXTREMITY INJURIES AMONG CONTEMPORARY DANCE STUDENTS}

${ }^{1}$ Christine van Seters, ${ }^{2,3}$ Rogier van Rijn*, ${ }^{1}$ Marienke van Middelkoop, ${ }^{2,3}$ Janine Stubbe. ${ }^{1}$ Department of General Practice, Erasmus MC University Medical Center, Netherlands; ${ }^{2}$ Codarts University of the Arts, Netherlands; ${ }^{3}$ Performing artist and Athlete Research Lab (PEARL), Netherlands

\subsection{6/bjsports-2019-scandinavianabs.31}

Introduction Contemporary dance students are at high risk for injuries; a yearly overall risk of $\geq 60 \%$ with lower-extremity injuries as the most predominant musculoskeletal injuries. Therefore, the objective was to determine whether student characteristics, lower-extremity kinematics, and strength are risk factors for sustaining lower-extremity injuries in preprofessional contemporary dancers.

Materials and methods A prospective cohort study with 45 1 year students of Bachelor Dance and Dance Teacher was set up. At the beginning of the academic year, injury history (only lower-extremity) and student characteristics (age, sex, educational program) were assessed using a questionnaire. Lower-extremity kinematics [single-leg squat(SLS)], strength (countermovement jump) and height and weight were measured during a physical performance test. Substantial lowerextremity injuries (main outcome) during the academic year were defined as any problems leading to moderate/severe reductions in training volume/performance, or complete inability to participate in dance at least once during follow-up as measured with the Oslo Sports Trauma Research Center (OSTRC) Questionnaire. Injuries were recorded on a monthly basis using a questionnaire. Analyses on leg-level were performed using generalized estimating equations(GEE) to test the associations between substantial lower-extremity injuries and potential risk factors.

Results The 1 year incidence of lower-extremity injuries was $82.2 \%$. Of these, $51.4 \%$ was a substantial lower-extremity injury. Multivariate analyses identified that ankle dorsiflexion during the SLS (OR1.25;95\% CI,1.03-1.52) was a risk factor for a substantial lower-extremity injury.

Conclusions The findings indicate that contemporary dance students are at high risk for LE injuries. Therefore, the identified risk factor (ankle dorsiflexion) should be considered for prevention purposes.

\section{TO INVESTIGATE FEASIBILITY OF A BANDCIZERTM DEVICE TO EVALUATE ADHERENCE TO HOME EXERCISE (HEP) FOR ADULTS WITH SHOULDER PAIN}

${ }^{1}$ Christina O'Connor, ${ }^{1}$ Jamie McNamara, ${ }^{1}$ Kathryn Fahy, ${ }^{1}$ Niamh Cleary, ${ }^{1}$ Paul Deering,

${ }^{1,2}$ Karen McCreesh*. ${ }^{1}$ School of Allied Health, University of Limerick, Ireland; ${ }^{2}$ Health Research Institute, University of Limerick, Ireland

\subsection{6/bjsports-2019-scandinavianabs.32}

Introduction Exercise is an effective intervention for rotator cuff related shoulder pain (RCRSP). Self-report methods of exercise adherence are subject to bias. The BandCizer ${ }^{\mathrm{TM}}$ is a 